\title{
Role of viruses in oral squamous cell carcinoma
}

\author{
Rashmi Metgud, Madhusudan Astekar, Meenal Verma, Ashish Sharma \\ Department of Oral and Maxillofacial Pathology, Pacific Dental College and Hospital, \\ Debari, Udaipur (Rajasthan), India
}

\begin{abstract}
The etiology of oral squamous cell carcinoma (OSCC) is complex and involves many factors. The most clearly defined risk factors are smoking and alcohol, which substantially increase the risk of oral SCC. However, despite this clear association, a substantial proportion of patients develop OSCC without exposure to them, emphasizing the role of other risk factors such as genetic susceptibility and oncogenic viruses. Some viruses are strongly associated with OSCC while the association of others is less frequent and may depend on co-factors for their carcinogenic effects. Therefore, the exact role of viruses must be evaluated with care in order to improve the diagnosis and treatment of OSCC.
\end{abstract}

\section{Introduction}

Cancer of the oral cavity is the sixth most common malignancy worldwide and accounts for approximately $5 \%$ of malignant tumors in developed countries. ${ }^{1}$ There has been a steady rise in incidence, especially because of the emergence and strong hold taken by the dreaded acquired immunodeficiency syndrome (AIDS). Our life style and environment are also having a big effect on our biological system, and these have both a direct and indirect role in the causation of cancer.

Correspondence: Madhusudan Astekar, Department of Oral and Maxillofacial Pathology, PAHER University, Pacific Dental College and Hospital, Debari, Udaipur (Rajasthan) - 313024, India.

Mob: +91.9413026974. E-mail: madhu.tanu@gmail.com

Key words: cellular proteins, viral genome, transforming region, apoptosis, caspases, transforming genes, oncogene proteins, squamous cell carcinoma, AIDS, HPV, EBV, vaccines.

Conflict of interests: the authors declare no potential conflict of interests.

Acknowledgements: we would like to acknowledge all the staff members in the department of oral and maxillofacial pathology for their support.

Received for publication: 28 April 2012.

Revision received: 3 September 2012.

Accepted for publication: 4 September 2012.

This work is licensed under a Creative Commons Attribution NonCommercial 3.0 License (CC BY-NC 3.0).

(C) Copyright R. Metgud et al., 2012

Licensee PAGEPress, Italy

Oncology Reviews 2012; 6:e21

doi:10.4081/oncol.2012.e21
Oral squamous cell carcinomas (OSCC) usually originate from the non-keratinizing stratified mucosal epithelium and show morphological similarity to squamous cell carcinomas of other body regions, like those of cervix, anus, or bronchi. It is generally agreed that tobacco, betel quid, and alcohol consumption are the major environmental risk factors for developing OSCC.,3 However, some patients develop OSCC without exposure to these risk factors. This element suggests that additional causes, such as genetic predisposition, diet, or oncogenic viruses, may also help cells to override or escape the physiological mechanisms of proliferation control. ${ }^{2}$ If we look back, the fact that has best stood the test of time is that non-lethal genetic damage lies at the heart of carcinogenesis. Almost 100 years ago, Peyton Rous established that viruses can cause cancer by demonstrating that chicken sarcomas could be transmitted by a virus called Rous Sarcoma Virus. In the 1930s, it became apparent that tumors in rabbits could be transmitted by a virus: Shope Papillomavirus. ${ }^{4,5}$

\section{Establishing an infectious etiology of oral squamous cell carcinomas}

Incidence and prevalence of OSCC is significantly higher in subjects exposed to the putative virus than in those not exposed. Evidence of exposure to the putative virus is more common in subjects with the cancer than in those without. Temporarily, the onset of the cancer follows exposure to the putative virus, and a spectrum of signs and symptoms follows such exposure. A measurable host response, such as antibody response and/or a cell-mediated immune response, follows exposure to the putative virus. Experimental reproduction of the cancer follows deliberate exposure of animals or humans to the putative virus but non-exposed control subjects remain disease free. Elimination of the putative virus and/or its vector decreases the incidence of the cancer. Prevention or modification of infection via immunization or drugs reduces the incidence of the cancer and, finally, the whole concept should follow biological and epidemiological reasoning. ${ }^{6}$

\section{Viruses strongly associated with oral squamous cell carcinomas}

\section{Human papilloma viruses}

They belong to a group of small double stranded DNA oncoviruses. Because of their superficial similarity in electron microscopic appearance and biological properties, they were originally classified as members of the Papovavirus family along with mouse polyoma virus and simian vacuolating virus (SV40). Recent molecular and genetic studies have revealed differences from the Papovavirus family, such as larger DNA genome, larger capsid, different open reading frames, and 
pattern of RNA synthesis. Finally, in 1991, Chang et al. considered them as a distinct and unique virus family. ${ }^{?}$

\section{Classification}

Papillomaviruses are classified according to their host range and the relatedness of their nucleic acids.

i. Papillomavirus was first named according to its natural host, e.g. Cottontail Rabbit (Shope) Papillomavirus, Bovine Papillomavirus, Deer Papillomavirus, Human Papillomavirus (HPV), etc.

ii. Based on clinical prognosis of their associated lesion they can be: low-risk HPVs, which cause benign epithelial hyperplasia, and high-risk HPVs, e.g. HPV 16 and 18 infected lesions have high propensity for malignant transformation.

iii. According to the International Agency for Research on Cancer: ${ }^{8}$ Group 1: HPV 16 and 18 as carcinogenic in humans; Group 2A: HPV 31 and 33 as probably carcinogenic in humans; Group 2B: remaining HPVs as possibly carcinogenic.

\section{Structure of human papilloma virus}

Human papillomaviruses are a DNA virus family of approximately 200 types that display a marked tropism for squamous epithelium. Despite a similar genomic make-up, different HPVs infect epithelia at distinct anatomic locations. Approximately $30 \mathrm{HPV}$ types infect the anogenital and oral mucosa that can be further classified as low-risk and high-risk based on the clinical prognosis of their associated lesions. Virtually all cervical carcinoma cases are associated with highrisk HPV infection, and two viral proteins, E6 and E7, which are consistently expressed in the tumors, are required for both the induction and maintenance of the transformed phenotype. ${ }^{9}$ Open reading frame regions are divided into early (E) and late (L) regions. E regions, designated as E1-E7, are expressed soon after infection and encode proteins involved in the induction and regulation of viral DNA replication. Out of these early proteins E6, E7 and E5 proteins are important. L regions, designated as L1 and L2, are expressed later in the infection and encode viral capsid proteins.

\section{Link between high-risk human papilloma viruses, cell cycle and oral squamous cell carcinomas}

In a normal keratinocyte, DNA damage induces p53 expression that in turn up-regulates p21 expression. P21 binds with proliferative cell nuclear antigen (PCNA), deactivates it and thus blocks the phosphorylation of the retinoblastoma gene (pRb). Thus hypo-phosphorylated $\mathrm{pRb}$ binds and deactivates E2f, a transcription factor. PCNA and E2f inactivity inhibits DNA replication and oral keratinocyte division. DNA damage may result in apoptosis of oral keratinocyte. ${ }^{10}$

The relevance of the role of HPV infection in cervical and anal cancer is well established and, by way of analogy, oncogenic HPV viruses might play a role in malignant transformation of squamous epithelia in any body region. ${ }^{11}$ Although still controversial, such evidence implies that carcinogenesis by environmental chemical carcinogens is not the only cause of OSCC.

Syrjanen et al. were the first to provide, in 1983, evidence on HPV as an etiological factor in OSCC when cytopathic effects of HPV (koilocytosis) were noted on light microscopy of oral carcinomas and the presence of HPV antigens in 40 oral carcinomas were analyzed using immunohistochemistry. Of the 40 lesions, 16 (40\%) showed HPV suggestive changes on light microscopy, and of those, 8 of 16 expressed HPV structural proteins. ${ }^{12}$ A few years later in 1990, these biopsies were examined by Chang et al. for the presence of HPV DNA using in situ hybridization (ISH) and polymerase chain reaction (PCR), and 12 of 40 disclosed the presence of HPV 11, 16 or 18 DNA. $^{7}$

Presence of HPV in variable proportions in the oral or oro-pharyngeal squamous cell carcinoma tissues other than the uterine cervix, espe- cially those genotypes with known high oncogenic potential (such as HPV 16 and 18) has been demonstrated by several worldwide studies ${ }^{7-}$

${ }^{11}$ but the data available are highly inconsistent. The wide differences in the results have been attributed to:

site of sampling, since HPV infection is significantly more frequent in oro-pharyngeal mucosa compared with oral mucosa. The difference strongly suggests anatomical site specificity. It also implies that the evidences of HPV in mucosal tissue of head and neck other than oral cavity are far more easily detected. The motile nature of the oral cavity, along with saliva secretion and cleaning, is perhaps at least in part responsible for the lower detection rate of HPV. In the oral cavity, a part of the oral mucosa is heavily keratinized, similar to the skin, and the number of nucleated cells might remain very low, depending on the site of the swab sampling;

patient populations, which might differ in terms of rates of endemic infections and HPV types, as well as in terms of lifestyle, such as sexual habits, tobacco smoking, alcohol consumption, etc.;

different samples;

difference in methodology. A problem with using PCR is the possibility of false positivity due to the amplification of human DNA or laboratory contamination;

difference in sample size. Analysis of HPV is suggested in normal mucosa of healthy patients as compared to its detection in neoplastic lesions. Therefore, standardization of the methods for sample collection and analysis is mandatory to obtain reliable data and to allow the results obtained in different studies to be compared..$^{13}$

Up till 1997, a total of 2380 oral carcinomas had been analyzed for HPV DNA using different techniques, and 31\% (746 of 2380) were reported to contain HPV. Between 1998 and 2002, 19 additional studies reported an analysis of 2388 oral carcinomas, of which $12.4 \%$ were HPV DNA-positive (295 of 2388). Five studies have detected HPV DNA in both the primary tumor and its lymph node metastases (16 of 21) cases. ${ }^{14}$ Between 1984 and 2004, 271 cancer tissue samples collected from oropharyngeal cancer patients in Hawaii, Iowa and Los Angeles were tested. In 1984-89, approximately16\% of oropharyngeal cancers (cancers of the tonsils, upper throat and base of the tongue) tested HPV-positive; by 2000-04, the proportion of HPV-positive cancers had risen to $72 \%$ which means that the rate of HPV-related oral cancer rose from 0.8 cases per 100,000 of the population in the 1980 s to 2.6 cases per 100,000 in the $2000 \mathrm{~s}$; an increase of $225 \%$. At the same time, the study found that as cigarette smoking declined, there was a 50\% drop in the rate of HPV-negative cancers. Overall, the risk was greatest and rising in men, and the findings suggest that it may have to do with an increase in oral sex. Meredith Melnick believed that sexual habits have changed, and that there is an increase in sexual activity earlier on in life, with an exchange of many more sex partners in general. Similar views have been expressed in recent studies. ${ }^{15,16} \mathrm{HPV}$ is best known as the virus that causes cervical cancer in women, but because of better screening, the rate of such cancers has declined over time. The authors predict that if current trends continue, oral cancers may become the most common HPV-related cancer by 2020 , eclipsing cervical cancer. Also, the burden of invasive HPV-caused cancers in the US will shift from women to men, largely due to the rise of HPV-positive oropharynx cancers among men. The good news is that patients with HPV-positive oral cancers have better survival rates than those with cancer due to other causes, possibly because their tumors cause less genetic damage, which makes them more responsive to treatment. ${ }^{17}$

In 2005, Syrjanen suggested, after 20 years of active research on the subject, that their conclusions from the early 1980s, that HPV seems to be associated with at least a proportion of oral carcinomas, are still valid. ${ }^{13}$ A study by Sisk et al. showed no significant difference in the incidence of HPV between younger patients and older patients, suggesting a similar role for HPV in all age groups. ${ }^{14}$ 
A large volume of research ${ }^{18,19}$ has revealed many details of how HPVs induce and maintain the malignant phenotype. Two proteins of the virus are particularly important: E6 and E7. The E6 protein has the ability to specifically bind to the cellular p 53 protein, and this leads to the breakdown of p53 and loss of its concentration in cancer cells. The results of this are numerous, including the lack of DNA repair following damage by other agents and lack of ability to die by apoptosis. The E7 protein can bind to the cellular Rb protein, releasing transcription factors such as E2f that are then free to transactivate the expression of other cellular proteins. The net effect of the papillomavirus infection is, therefore, a series of changes in the cell that combine to appear as malignant phenotype..$^{20}$ Sugarman and Shillitoe critically evaluated and simplified the association between HPV and OSCC and postulated that E6 protein insertion alone into the keratinocyte is not sufficient to cause oral cancer and some additional growth promoting factors (chemical carcinogen) are required for cancer development. E7 alone can cause cancer but p53 would detect the DNA involving E7 insertion and would block cell division. For E7 to cause cancer there should also be simultaneous reduction in p53 activity. Therefore, it was finally concluded that a combination of high-risk HPV and chemical carcinogen is a potentially dangerous cocktail and, secondly, that simultaneous activity of E6 and E7 within a single keratinocyte is essential for uninhibited PCNA and E2f activity. ${ }^{10}$

Although it has been suggested that high-risk HPV may induce some percentage of oral carcinogenesis, more evidence is now emerging that HPV infection may also have the potential to significantly alter oral cancer proliferative phenotypes and outcomes. Determining the potential of HPV to alter phenotypic behaviors of already transformed oral carcinomas has, therefore, become an important step in determining more accurately the prognosis and treatment options for patients with oral cancer. In 2006, Karl Kingsley ${ }^{21}$ determined that the OSCC cell line, CAL27, transfected with HPV16, exhibited significantly increased proliferation when compared with non-transfected controls. This increased proliferation was observed even in the absence of serum, and the effects were specific to proliferation, adhesion, and morphology, but not to cell viability. In 2007, Reddout $e t$ al. investigated the role of HPV in already transformed OSCC and suggested that CAL27 cells transfected with HPV18, HPV16, as well as HPV16/18 co-transfectants, demonstrated significant increases in proliferation, adhesion and cell spreading compared with non-transfected controls. These observed differences were correlated with a small level of increased cell survival. SCC-15 cells, however, displayed a differential response to HPV transfection, with only HPV18-transfectants demonstrating changes to proliferation. Interestingly, SCC-25 cells displayed a more complex response, with HPV16-induced increases in cell proliferation, viability and cell spreading, while HPV18- and 16/18-transfectants exhibited reduced adhesion and proliferation. The identification of differential responses to specific HPV strains among oral cancers suggests a more significant, complex and multifactorial role of HPV, not only in transforming, but also in modulating the phenotype and treatment responsiveness of pre-cancerous and cancerous oral lesions. ${ }^{22}$

In a study by Min Dai et al., TP53 mutations were found more frequently in oral cancer specimens from smokers than non-smokers, the presence of HPV16 DNA was, in a few studies, more frequent among non-smokers than smokers. This is, perhaps, not surprising because at least some high-risk HPV types and chemical carcinogens can inactivate p53 suppressor function and thus can be considered alternative pathways to chromosomal instability, uncontrolled proliferation, and malignant transformation in precursors of oral cavity cancers. Their findings, however, suggest that, for the relationship between TP53 mutations and HPV16 infection to be mutually exclusive, it is not sufficient to find HPV DNA in cancer specimens, but some marker of E6 expression must also be identified. In other words, the ability of HPV16 DNA to predict wild-type TP53 (69\%) is substantially improved (to
$100 \%)$ by taking into consideration information on the presence of HPV16 E6 antibodies. High-risk HPV types, however, can promote carcinogenesis through mechanisms other than inactivation of p53, such as via the interaction of the viral protein $\mathrm{E} 7$ with $\mathrm{pRb}$, the product of the retinoblastoma suppressor gene RB1. Therefore, we exercise caution before denying a role for HPV in cancers containing TP53 mutations, even more so because the vast majority of cancers of the oral cavity, including those positive for HPV DNA, are presented in smokers or chewers. $^{23}$

Considering that the OSCC is the second most common HPV associated cancer, and since its incidence is increasing, the effect of HPV vaccine on this tumor deserves attention. ${ }^{24}$ Jalouli $^{25}$ investigated the prevalence of virus in pre-malignant and malignant samples in three different populations (India, Sudan, Sweden) exposed to three different types of non-smoking tobacco habits: betel quid chewing; the snuff dipping habit called toombak; and the use of snuff.

There was an observed higher incidence of virus infection with human papilloma virus and Epstein Barr virus in potentially malignant than in malignant lesions; approximately $29 \%$ in leukoplakias and lichen planus from Sweden, 40\% of the toombak users in Sudan, and particularly high yields (91\%) of HPV DNA in oral submucous fibrosis from India.The author concluded that the study data do not at first sight support the conclusion that viruses and tobacco use jointly interact with cell mechanisms in the development of oral cancer.

Author's data from a study by Su Feng et al. revealed 24 of 65 cases of OSCC showing evidence of HPV by ISH. Among these 24 positive cases, 14 were found positive for p16INK4A via immunohistochemistry. ISH for evidence of HPV infection in OSCC appeared more sensitive than immunohistochemical detection of p16INK4A. The majority (37 of 41) of HPV-negative cases of OSCC were related to overexpression of p53. In addition to 11 cases co-expressed with ISH for HPV and p53, there were a total of 48 cases of OSCC showing overexpression of p53 (48 of 65) providing strong evidence that HPV is etiologically linked to a defined subset of OSCC. Compared with previous reports in which different methods conclude that the HPV-positive rate of OSCC ranges from $43 \%$ to $75 \%$, the author's data with a rate of nearly $37 \%$ HPV positive seems obviously lower than those in oropharynx and pharynx. The Authors concluded that the motile nature of the oral cavity along with saliva secretion and cleaning could perhaps, at least in part, be responsible for the lower detection rate of HPV. ${ }^{11}$

\section{Herpes simplex virus}

Gene maps of herpes simplex virus (HSV) 1 and 2 have been developed and show that the various functions of the two viruses are encoded by identical regions of the genome of each virus. A comparison of the two viruses showed that the only viral function that is not in a related location is the ability to transform cells: the transforming region of HSV 1, minimum transforming region [mtr]-1, is located in the left third of the genome, while in HSV 2, mtr-2, mtr-3 are close to the center of the genome. ${ }^{26}$

\section{Role of herpes simplex virus in animal and human models}

The first animal model that demonstrated that HSV could be a co-carcinogen was introduced by Southam et al. Before this it was known that if low doses of the hydrocarbon carcinogen methylcholanthrene were applied to the skin of mice, a low proportion of them would develop skin tumors. Later, Southam et al. showed that an infection with HSV-1 could increase the proportion of animals with pre-malignant lesions from $29 \%$ to $51 \%$ and increase the proportion with carcinomas from $7 \%$ 
to $19 \%{ }^{27}$ A second animal model was introduced by Duff and Rapp, working initially with HSV-2 but later with HSV-1. They showed that if either virus was partially inactivated with various agents so as to prevent it from killing cells, a certain number of the surviving cells would then transform to a malignant phenotype. The cells were found to be extremely aggressive, and would not only invade local tissue but also metastasize to distant sites in the animal setting up secondary tumors in liver and lungs. ${ }^{28}$

A situation more similar to that in humans would be one in which HSV-1 becomes latent in the trigeminal ganglion after which the oral mucosa is exposed to carcinogens. This has now been reproduced in animals. Hamsters were exposed to intra-oral infections with HSV-1 and, after they had recovered, the cheek pouches were exposed to 7,12dimethyl-benz[a]anthracene (DMBA). The number of tumors that developed per pouch was significantly higher in those animals that had been previously infected with HSV-1; their mucosa showed more severe histological changes, and the expression of the erb-B oncogene was also increased. Interestingly, when the trigeminal ganglia were examined it was found that in the animals given DMBA plus HSV-1, 95\% had detectable latent virus, while in the absence of DMBA only $10 \%$ of animals showed latent virus. The significance of this is unclear, but intriguing. Perhaps one effect of carcinogens is to promote the maintenance or replication of virus in ganglia. Levels of antibody to HSV-1 in the animals were not measured but might be expected to be raised due to over-exposure to the virus. ${ }^{29}$ If this is the case, then the situation would be similar to the observation in humans that cigarette smokers have higher levels of antibody to HSV-1 than non-smokers. ${ }^{30}$

The simultaneous exposure of oral mucosa to HSV-1 and tobacco products was first reproduced in rats by Hirsch et al. They developed an ingenious system in which a surgical procedure was used to make a canal inside the lower lip in which a wad of chewing tobacco could be placed. This would be retained for several days, after which it could be replaced. Some animals were also inoculated with HSV-1, and it was found that exposure to both the tobacco and the virus produced more tumors than exposure to either agent alone. The mechanism behind the interaction is not known, but it has been found that extracts of chewing tobacco inhibit the ability of HSV-1 to kill cells. ${ }^{31}$ Thus, the mechanism might resemble the cell transformation model introduced by Rapp. ${ }^{28}$ The alternative explanation is that the HSV-1 potentiates tumors that are induced by carcinogens in the tobacco. However, since there are no specific cell markers that distinguish HSV-induced or tobacco-induced tumors the question will be hard to resolve. Interestingly, animals exposed to HSV-1 and tobacco, or HSV-1 and chemical carcinogens have an increased incidence of tumors at extraoral sites as well as oral tumors, although no explanation for this is available. $^{32}$

Patients with oral cancer have generally been exposed to HSV, as judged by the fact that most have antibodies to the virus. An earlier study showed that although their overall level of neutralizing antibody is similar to that of matched control subjects, some differences in the antibodies are seen; ${ }^{33}$ the patients have higher levels of IgA antibodies to a protein present in cells that are infected by the virus and higher levels of IgM antibody to an antigen present on the virus particle. The viral antigens that are recognized by the patients' sera have not been identified. The tumors do not express the major antigens of HSV, but since the virus encodes some 80 proteins it has not yet been possible to test sera for antibody to all possible antigens. ${ }^{32}$ Early studies attempted to verify whether DNA of HSV could be detected in oral cancers, and preliminary reports did indicate the presence of both/either viral DNA and/or RNA. ${ }^{34}$

The role of herpes simplex viruses, HSV-1 and HSV-2, as co-factors in association with tobacco, alcohol, or HPV-16 infection has also been examined. Heavy use of tobacco, alcohol and HPV-16 infection was associated with an increased risk of OSCC but, after adjusting for age, tobacco, alcohol use, and number of sexual partners, the risk of cancer was not significantly increased in those with HSV-1 or HSV-2, though seropositivity to HSV-1 and HSV-2 may modify the risk associated with exposure to tobacco, alcohol or HPV. ${ }^{35}$

Recent years have seen little or no progress in the study of HSV and its malignant potential. ${ }^{17}$

\section{Role of herpes simplex virus in cell transformation}

The transforming mechanisms of HSV 1 and 2 remain obscure. The viruses have not been shown to encode an oncogene or related gene and furthermore the transforming regions of the genome are not retained in transformed cells. Galloway et al. narrowed down the transforming region of HSV 2, mtr-2, to 793 base pairs of DNA. ${ }^{36}$ Even such a small amount of viral DNA was not retained by the transformed cells, leading to the so-called hit and run hypothesis of transformation by HSV proposed by Ambinder to be widely adopted. ${ }^{37}$ At one time it was suggested that the sequence contained an insertion sequence-like element, although no experimental evidence for such an activity has emerged, and the hypothesis was not supported by a later computerized sequence analysis. The sequence does, however, contain the carboxy terminus of an open reading frame. The product of this reading frame of HSV 2 is unknown and has not been investigated. ${ }^{32}$

Ambinder's proposed hit and run scenario of oncogenesis is based on observations that viral episomes can be completely lost from certain cell lines. According to this theory, transient acquisition of a complete or incomplete viral genome may be sufficient to induce malignant conversion of host cells in vivo, resulting in neoplastic development. After eliciting a heritable change in the gene expression pattern of the host cell, the genomes of tumor viruses may be completely lost. ${ }^{37}$

Several mechanisms of cell transformation by HSV have been proposed. ${ }^{32}$

Transforming regions of the genome of HSV: comparisons between the sequence of mtr-2 of HSV-2 and the equivalent but non-transforming region of HSV 1 have revealed some differences, but they have only been studied with respect to the likelihood of secondary structure formation. No study has compared the sequence of the encoded proteins between the two viruses or compared the sequence of the transforming region of HSV 1 with that of HSV 2. Therefore, the important similarities and differences between the protein products of the transforming regions of the two viruses are not known. The only apparent similarity is the recent observation that the two protein products are similar given that a computer search of the protein data bank revealed that each can be aligned with the EBNA-1 gene (Epstein Bar Nuclear Antigen-1) of Epstein-Barr virus. In HSV 2, there is another transforming region, known as mtr-3, which encodes the ribonucleotide reductase gene of the virus. This gene has been proposed to be a transforming gene of that virus but it is not retained by the transformed cells and has not gained acceptance as a transforming gene. There has been no evidence that HSV-1 might use this enzyme in cell transformation. ${ }^{38}$

Mutagenesis by HSV: attention has recently been turned to the possibility that HSV transforms cells by acting as a mutagen. Earlier studies demonstrated chromosomal aberrations in HSV infected cells that are typical of the effects of a mutagen. Studies of the frequency of mutations at the hypoxanthine guanine ribosyl transferase locus of mammalian cell genomic DNA showed an increase in mutation frequency of up to 2-4 fold following infection by HSV 1 that had been inactivated by UV light. ${ }^{29}$ The same phenomenon has been observed by Pilon et al., in cells infected by HSV $2 .{ }^{39}$ In 1991, Steele and Shillitoe ${ }^{32}$ suggested that HSV 1 expresses a viral protein designated as MUT. Other studies related MUT to the p40 protein family present in nucleus of infected cells, but the function of this family protein was not described. Based on these studies, Steele and Shillitoe hypothesized that the p40 family or 
the MUT protein could be involved in host cell shut off. ${ }^{29}$ Mutant DNA mismatch repair proteins recognize and repair erroneous insertions, deletions and mis-incorporation of bases that may arise during DNA replication and recombination. Mutations in this lead to genomic changes by causing microsatellite instability that has been implicated in malignancy. Some HSV 1 induced mutant plasmids contained rearranged chromosomal DNA which are typical of cancers associated with DNA tumor viruses. So it was concluded that chromosomal rearrangements by HSV might be present in tumors that are associated with the virus and should be detectable by the appropriate probes. ${ }^{40}$

\section{Other mechanisms}

Induction of cellular proteins: infection by HSV induces the expression of stress or heat shock proteins. According to Steele and Shillitoe, the exact mechanism is not known but it depends on the expression of the immediate early family of HSV proteins. ${ }^{32}$ It was, therefore, concluded that HSV might transform cells by stimulating the expression of cellular proteins. ${ }^{41,42}$

Host cell shut off process: infected cell ceases to synthesize cellular proteins and cell RNA would be very quickly degraded. A mediating gene was located in the same region of the genome as the mtr-2 region of HSV 2 that mediates cell transformation. ${ }^{43}$ Steele and Shillitoe raised the possibility that the mechanism of cell transformation might be related to the mechanism of shut off. ${ }^{32}$

Stimulation of other viruses by HSV: this comes from the idea that at least in cervical carcinogenesis, HSV and HPV may act as co-carcinogens, with HSV as an initiator and HPV as a promoter. In oral lesions, however, Scully et al. in 1993 showed that very few pre-malignant or carcinoma specimens appeared to have both HPV 16 and HSV 1 DNA sequences. They, therefore, concluded that there is no evidence that these potentially oncogenic DNA viruses do play a synergistic role in oral cancer development, but the possibility cannot be discounted. ${ }^{44}$

Chromosomes as targets: Stich et al., Mincheva et al. and Peat suggested that when cells are infected by HSV there is chromosomal damage which is at first restricted to a site on chromosome 1q and to some extent on chromosome 3, 9 and 16. Based on these studies, Steele and Shillitoe gave a plausible explanation for the possibility that HSV has specific chromosomal targets for rearrangement. Damage to a particular chromosomal site might be another possible mechanism of cell transformation by HSV. ${ }^{45,46,47,32}$

\section{Viruses less frequently associated with oral squamous cell carcinoma}

\section{Epstein-Barr virus}

Epstein-Barr virus (EBV) is named after Michael Anthony Epstein and Yvonne Barr, who discovered and documented the virus in 1964. $\mathrm{EBV}$, also called human herpes virus 4 (HHV-4), is a virus of the herpes family. The virus consists of a linear double-stranded DNA core surrounded by a nucleocapsid and an envelope that contains glycoproteins. EBV is a ubiquitous human gamma-herpes virus that is commonly associated with a number of malignancies such as Burkitt's lymphoma, Hodgkin's disease, stomach carcinomas and nasopharyngeal carcinoma (NPC). ${ }^{48}$

\section{Epstein-Barr virus and oral squamous cell carcinoma}

Keisuke et al. carried out a study to determine the presence of EBV in various squamous cell proliferative lesions in the oral cavity. ${ }^{49}$ They made use of PCR and in situ hybridization for detecting the presence of EBV DNA and EBV encoded small messenger RNA. Nearly $60 \%$ of
SCC was EBV genome positive, but none of papilloma demonstrated EBV genome. On the other hand, oral hairy leukoplakia lesion seen in patients with AIDS has been proved to be EBV-associated. Keisuke $e$ t al. concluded that EBV virus infection of oral squamous epithelium may be carcinogenic or, alternatively, the virus may merely exist in epithelial cells of squamous cell carcinoma, carcinoma in situ and leukoplakia. ${ }^{49}$ González-Moles et al. showed a positive correlation between different grades of OSCC and EBV DNA positivity, and also showed that percentage positivity of EBV DNA increases from well differentiated OSCC to poorly differentiated OSCC..$^{0}$

In a study by Higa et al., fifty-four patients with oral squamous cell carcinoma reported from 1997 to 1999 in Okinawa were compared with 21 and 20 patients from Kitakyushu and Kumamoto in Kyushu, mainland Japan, respectively. ${ }^{51}$ Diagnosis was confirmed by conventional histological examination of paraffin wax sections. EBV was detected by non-isotopic in situ hybridization and PCR [Bam HI-F, EBV nuclear antigen 2 (EBNA2), and latent membrane protein 1 (LMP-1) regions]. Sequence analysis of the PCR products was also carried out. In Okinawa, 25 patients were found to be infected with EBV type A by analyzing the 3 sequence divergence of the EBNA2 genes. Six patients were positive for EBV type B, and 8 for both type A and B. Therefore, type A virus infection was demonstrated in 33 of 54 patients, and type $\mathrm{B}$ in 14 of 54 . In total, 39 of 54 patients were infected with EBV. Authors concluded that in Okinawa, EBV infection was frequently demonstrated in oral squamous cell carcinoma $(\mathrm{P}<0.001)$. However, in mainland Japan, there was no significant correlation between EBV and oral squamous cell carcinoma. ${ }^{51}$

A study by Ching-Yu Yen et al. to detect EBV infection and gene expression in oral cancer from patients in Taiwan by microarray analysis revealed that the majority of the specimens (82.5\%) were EBV-positive that probably expressed coincidently the genes for EBNAs, LMP2A and $2 \mathrm{~B}$, and certain structural proteins. Importantly, the genes fabricated at the spots 61 (BBRF1, BBRF2, and BBRF3) and 68 (BDLF4 and BDRF1) on EBV-chip were actively expressed in a significantly greater number of OSCC exhibiting exophytic morphology or ulceration than those tissues with deep invasive lesions. ${ }^{52}$

In a study conducted by Sand et al. examining 29 patients with OSCC, 23 with OLP, and 67 with clinically healthy oral mucosa, a nested polymerase chain reaction method for EBV DNA analysis was used. The overall EBV prevalence in patients with oral disease was $32.1 \%$. Of the OSCC patients, $37.9 \%$ were EBV positive; and of the OLP patients, 26.1\% were EBV-positive. Both percentages were statistically significant compared with that of control patients (7.3\%). The difference in EBV prevalence between the smoking control group and the non-smoking control group was without significance. Increased age did not enhance EBV prevalence. The Authors were of the opinion that EBV is present in oral diseases such as OSCC and OLP. Smoking, alcohol use, or age does not seem to be a risk factor for EBV infection. ${ }^{53}$

Shimakage et al. showed that in situ hybridization using a probe comprising the transcripts of the BamHIW fragment of the EBV genome demonstrated EBV mRNA in the majority of tumor cells in all cases of oral cancer, but none in normal tissues. RNA in situ hybridization using an EBER1 probe detected RNAs in 16 of 24 cancers. Also, mRNA in situ hybridization using a probe of the EBV-determined nuclear antigen-2 (EBNA2) region detected positive signals in 9 of 12 cancers. Furthermore, EBNA2, latent membrane protein-1 (LMP1) and BZLF1 were detected in these cancers by immunofluorescence staining, but were not detected in any of the epithelial cells of the normal tissues. Four of 6 metastatic tissues showed stronger fluorescence than that in the primary tissues. PCR detected the BamHIW sequence of EBV DNA in all cases, including the normal tissues tested. These findings indicate that EBV may be involved in neoplastic transformation in oral cancers, such as NPC. ${ }^{54}$ 


\section{Hepatitis $\mathrm{C}$ virus}

Hepatitis $\mathrm{C}$ virus (HCV), an RNA virus, is easily detectable in saliva of patients with chronic liver disease and is an etiological agent for most cases of non-A, non-B hepatitis, liver cirrhosis and hepatocellular carcinoma. Johnson et al. suggested a possible involvement of HCV in diseases outside the liver and concluded that since oral cavity is frequently exposed to $\mathrm{HCV}$ viruses, this in turn increases the risk of genetic instability in these cells. ${ }^{55}$ Nagoa et al. studied a group of 100 patients including $88 \mathrm{SCC}$. Anti-HCV antibodies were detected in sera of 25 patients. ${ }^{56}$ The exact mechanism is unclear. Gandolfo $\mathrm{S}$ et al. found very high prevalence of anti-HCV antibodies in patients with oral lichen planus (OLP).$^{57}$ Nagao et al. suggested that, since OLP is also histologically a disease of squamous cells, the squamous cells of oral region are continuously exposed to HCV from saliva as well as from serum in HCV-positive patients, and that this may be involved in the development of SCC and OLP in these patients. ${ }^{56}$

\section{Therapeutic possibilities}

If viruses like HSV are responsible for the development of oral cancer by a hit and run mechanism, prospects for specific treatment are not very good. Intervention might be possible if the effect of the virus had to be exerted over a long period on a pre-malignant lesion. The fact is that the specific inhibition of target genes can be achieved by expression of RNA molecules from the strand opposite the coding strand. ${ }^{37}$

It was first shown in cervical cancer cells that if viral RNA transcript was blocked by antisense RNA, the expression of the HPV gene could be suppressed, which may reduce the growth of cancer cells. Only one study has addressed the possibility of preventing growth of human cancer cells by expression of antisense E6/E7. Von Knebel Doeberitz et al. cloned E6 and part of E7 region of HPV 18 in a eukaryotic expression vector in the reverse orientation with respect to the promoter. ${ }^{58}$ Expression vector was introduced into C4-1 cells, a cervical cancer cell line that expresses HPV 18 genes. On expression of antisense E6/E7, the cells showed significant changes in the phenotype; the cell size and colony size were reduced both on plastic and in soft agar.

Simple antisense technology was later superseded by ribozymes. Ribozymes directed against E6/E7 transcribed patients are effective in reducing the growth rate of HPV containing cervical cancer HeLa cells. An added advantage is that ribozymes can cut the target RNA so that there is a permanent reduction in the pathological RNA. The most recent version of this therapy consists of siRNA, a molecule that also recognizes viral RNA targets and is also effective in silencing their expression..$^{59}$

A major advancement was the recent introduction of two vaccines for the prevention of HPV-associated diseases. These vaccines consist of virus-like particles that contain the L1 protein from several HPV types. Each vaccine has been tested in controlled clinical trials and is effective in reducing the incidences of cervical abnormalities. But their oral effects are yet not yet known. Currently these vaccines are recommended only in adolescent females. Their use in males and older females is still to be established. ${ }^{37}$

\section{Conclusions}

Although the etiology of squamous cell carcinoma of the oral mucosa involves many different agents, viruses are important. HPV-positive oral and oro-pharyngeal cancer makes up a distinct clinico-pathological entity. Standardization of the methods for sample collection and analysis are mandatory to obtain reliable data and to compare the results obtained in different studies on the presence of HPV in variable proportions in oral squamous cell carcinoma tissues. Some tumors are associated with papillomaviruses and some with viruses of the herpes family; however, the exact role of these viruses must still be evaluated carefully. These viruses may provide targets for therapy and for diagnostic tests, and may widen our understanding about the mechanisms by which the tumors develop.

\section{References}

1. Parkin DM, Bray F, Ferlay J, Pisani P. Global cancer statistics, 2002. Cancer J Clin 2005;55:74-108.

2. Nemes JA, Deli L, Nemes Z, Márton IJ. Expression of p16INK4A, $\mathrm{p} 53$, and $\mathrm{Rb}$ proteins are independent from the presence of human papillomavirus genes in oral squamous cell carcinoma. Oral Surg Oral Med Oral Pathol Oral Radiol Endod 2006;102:344-52.

3. Rautava J, Luukkaa M, Heikinheimo K, et al. Squamous cell carcinomas arising from different types of oral epithelia differ in their tumor and patient characteristics and survival. Oral Oncol 2007;43:911-9.

4. Rous P. A transmissible avian neoplasm. (Sarcoma of the common fowl) by Peyton Rous, M.D., Experimental Medicine for Sept. 1, 1910, vol. 12, pp.696-705. J Exp Med 1979;150:738-53.

5. Shope RE, EW. H. Infectious Papillomatosis of rabbits: with a note on the histopathology. J Exp Med 1933;58:607-24.

6. Fredericks DN, Relman DA. Sequence-based identification of microbial pathogens: a reconsideration of Koch's postulates. Clin Microbiol Rev 1996;9:18-33.

7. Chang F, Syrjänen S, Kellokoski J, Syrjänen K. Human papillomavirus [HPV] infections and their associations with oral disease. J Oral Pathol Med 1991;20:305-17.

8. IARC. Monographs on the evaluation of carcinogenic risks to humans. Human Papillomaviruses, vol. 90. Lyon: International Agency for Research on Cancer; 2007.

9. Khovidhunkit S0, Buajeeb W, Sanguansin S, et al. Detection of human papillomavirus in oral squamous cell carcinoma, leukoplakia and lichen planus in Thai patients. Asian Pac J Cancer Prev 2008;9:771-5.

10. Sugerman PB, Shillitoe EJ. The high risk human papillomaviruses and oral cancer: evidence for and against a causal relationship. Oral Dis 1997;3:130-47.

11. Chen SF, Yu FS, Chang YC, et al. Role of human papillomavirus infection in carcinogenesis of oral squamous cell carcinoma with evidences of prognostic association. J Oral Pathol Med 2012;41:915.

12. Syrjänen K, Syrjänen S, Lamberg $M$, et al. Morphological and immunohistochemical evidence suggesting human papillomavirus (HPV) involvement in oral squamous cell carcinogenesis. Int J Oral Surg 1983;12:418-24.

13. Syrjanen S. Human papillomavirus in head and neck cancer. J Clin Virol 2005;32 Suppl:S59-66.

14. Migaldi M, Pecorari M, Forbicini G, et al. Low prevalence of human papillomavirus infection in the healthy oral mucosa of a northern Italian population. J Oral Pathol Med 2012;41:16-20.

15. Anaya-Saavedra G, Ramirez-Amador V, Irigoyen-Camacho ME, et al. High association of human papillomavirus infection with oral cancer: a case-control study. Arch Med Res 2008;39:189-97.

16. D'Souza G, Agrawal Y, Halpern J, Bodison S, Gillison ML. Oral sexual behaviors associated with prevalent oral human papillomavirus infection. J Infect Dis 2009;199:1263-9. 
17. Melnick M. HPV linked to more oral cancers than smoking. TIME Healthland October 5, 2011. Available from: http:/healthland. time.com/2011/10/05/hpv-linked-to-more-oral-cancers-than-smoking/\#disqus_thread

18. Ha PK, Califano JA. The role of human papillomavirus in oral carcinogenesis. Crit Rev Oral Biol Med 2004;15:188-96.

19. Nair S, Pillai MR. Human papillomavirus and disease mechanisms: relevance to oral and cervical cancers. Oral Dis 2005;11:350-9.

20. Shillitoe EJ. The role of viruses in squamous cell carcinoma of the oropharyngeal mucosa. Oral Oncol 2009;45:351-5.

21. Kingsley K, Johnson D, O'Malley S. Transfection of oral squamous cell carcinoma with human papillomavirus- 16 induces proliferative and morphological changes in vitro. Cancer Cell Int 2006;6:14.

22. Reddout N, Christensen T, Bunnel A, et al. High risk HPV types 18 and 16 are potent modulators of oral squamous cell carcinoma phenotypes in vitro. Infect Agents Cancer 2007;2:1-11.

23. Dai M, Clifford GM, le Calvez F, et al. Cancer: matched analysis of the IARC multicenter study. Cancer Res 2004;64:468-71.

24. Ramqvist T, Dalianis T. Oropharangeal cancer epidemic and human papilliomavirus. Emerg Infect Dis 2010;16:1671-7.

25. Jalouli J. Human papilloma virus, Epstein-Barr virus, herpes simplex virus type-1 in oral squamous cell carcinoma from three populations. Series: Digital comprehensive summaries of Uppsala dissertations from the Faculty of Medicine; 578. Uppsala; Uppsala University: 2010. p 68.

26. Corey L, Spear PG. Infections with herpes simplex viruses. N Engl J Med 1986;314:686-91.

27. Southam CM, Tanaka S, Arata T, et al. Enhancement of responses to chemical carcinogens by nononcogenic viruses and antimetabolites. Prog Exp Tumor Res 1969;11:194-212.

28. Duff R, Rapp F. Oncogenic transformation of hamster cells after exposure to herpes simplex virus type 2. J Virol 1973;12:209-17.

29. Cornelia FA, Saper, CD, Christensen RE, Park NH. Effect of DMBA on oral cancer development in hamsters with latent HSV-1 infections in trigeminal ganglia. Oral Surg Oral Med Oral Pathol 1989;67:167-71.

30. Shillitoe EJ, Greenspan D, Greenspan JS, et al. Neutralizing antibody to herpes simplex virus type 1 in patients with oral cancer. Cancer 1982;49:2315-20.

31. Hirsch JM, Johansson SL, Vahlne A. Effect of snuff and herpes simplex virus 1 on rat oral mucosa. Possible association with development of squamous cell carcinoma. J Oral Pathol 1984;13:52-62.

32. Steele C, Shillitoe EJ. Viruses and oral cancer. Cri Rev Oral Biol Med 1991;2:153-75.

33. Das MC, Schantz SP, Shillitoe EJ. Antibody to a mutagenic peptide of herpes simplex virus in young adult patients with cancer of the head and neck. Oral Surg Oral Med Oral Pathol 1993;75:610-4.

34. Cox M, Maitland N, Scully C, et al. Human herpes simplex-1 and papillomavirus type 16 homologous DNA sequences in normal, potentially malignant and malignant oral mucosa. Eur J Cancer B Oral Oncol 1993;29B:215-9.

35. Parker TM, Smith EM, Ritchie JM, et al. Head and neck cancer associated with herpes simplex virus 1 and 2 and other risk factors. Oral Oncol 2006;42:288-96.

36. Galloway DA, Nelson JA, McDougall JK. Small fragments of herpes virus DNA with transforming activity contain insertion sequencelike structures. Proc Natl Acad Sci U S A 1984;81:4736-40.

37. Minarovits J, Banati F, Niller HH. Viral hit-and-run tumorigenesis. Future Virol 2011;6:13-5.

38. Huszar D, Bacchetti S. Is ribonucleotide reductase the transforming function of herpes simplex virus 2 ? Nature 1983;302:76-9.
39. Pilon L, Royal A, Langelier Y. Increased mutation frequency after Herpes simplex virus type 2 infection in non-permissive XC cells. J Gen Virol 1985;66:259-65.

40. Hwang CB, Shillitoe EJ. Analysis of complex mutations induced in cells by herpes simplex virus type-1. Virology 1991;181:620-9.

41. Filion M, Skup D, Suh M. Specific induction of cellular gene transcription in herpes simplex virus type 2-transformed cells. J Gen Virol 1988;69:2011-9.

42. La Thangue NB, Latchman DS. A cellular protein related to heatshock protein 90 accumulates during herpes simplex virus infection and is overexpressed in transformed cells. Exp Cell Res 1988;178:169-79.

43. Kwong AD, Kruper JA, Frenkel N. Herpes simplex virus virion host shutoff function. J Virol 1988;62:912-21.

44. Boyd AL, Enquist L, Vande Woude GF, Hampar B. Activation of mouse retro virus by Herpes simplex virus type 1 cloned DNA fragments. Virology 1980;103:228-31.

45. Stich HF, Hsu TC, Rapp F. Viruses and mammalian chromosomes. I. Localization of chromosome aberrations after infection with herpes simplex virus. Virology 1964;22:439-45.

46. Mincheva A, Dundarov S, Bradvarova I. Effects of herpes simplex virus strains on human fibroblast and lymphocyte chromosomes and the localization of chromosomal aberrations. Acta Virol 1984;28:97-106.

47. Peat DS, Stanley MA. Chromosome damage induced by herpes simplex virus type 1 in early infection. J Gen Virol 1986;67:2273-7.

48. Jain N, Bhatia V, Lattoo S. Epstein-Barr virus and associated head and neck manifestations. Ann Nigerian Med 2011;5:38-41.

49. Horiuchi K, Mishima K, Ichijima K, et al. Epstein-Barr virus in the proliferative diseases of squamous epithelium in the oral cavity. Oral Surg Oral Med Oral Pathol Oral Radiol Endod 1995;79:57-63.

50. Gonzalez-Moles M, Gutierrez J, Ruiz I, et al. EBV and OSCC in patients without HIV infection: viral detection by polymerase chain reaction. Microbios 1998;96:23-31.

51. Higa M. Epstein-Barr virus (EBV) subtype in EBV related oral squamous cell carcinomain Okinawa, a subtropical island in southern japan, compared with Kitakyushu and Kumamotoin mainland Japan. J Clin Pathol 2002;55:414-23.

52. Yen CY, Lu MC, Tzeng CC, et al. Detection of EBV infection and gene expression in oral cancer from patients in Taiwan by microarray analysis. Journal of Biomedicine and Biotechnology 2009;2009:904589.

53. Sand LP, Jalouli J, Larsson PA, Hirsch JM.. Prevalence of EpsteinBarr virus in oral squamous cell carcinoma, oral lichen planus, and normal oral mucosa. Oral Surg Oral Med Oral Pathol Oral Radiol Endod 2002;93:586-92.

55. Johnson RJ, Gretch DR, Yamabe H, et al. Membranoproliferative glomerulonephritis associated with hepatitis $\mathrm{C}$ virus infection. New Engl J Med 1993;328:465-70.

56. Nagao Y, Sata M, Itoh K, et al. Quantitative analysis of HCV RNA and genotype in patients with chronic hepatitis $\mathrm{C}$ accompanied by oral lichen planus. Eur J Clin Invest 1996;26:495-8.

57. Gandolfo S, Carbone M, Carrozzo M, Gallo V. Oral lichen planus and hepatitis $\mathrm{C}$ virus (HCV) infection: is there a relationship? A report of 10 cases. J Oral Pathol Med 1994;23:119-22.

58. Von Knebel Doeberitz M, Rittmuller C, zur Hausen H, Durst M. Inhibition of tumorigenicity of cervical cancer cells in nude mice by HPV E6/E7 anti-sense RNA [letter] Int J Cancer 1992;51:831-4.

59. Jiang M, Milner J. Selective silencing of viral gene expression in HPV-positive human cervical carcinoma cells treated with siRNA, aprimer of RNA interference. Oncogene 21:6041-48. 